Case Report

\title{
Minimally Invasive Treatment for Tibial Malrotation after Locked Intramedullary Nailing
}

\author{
Kyohei Takase $\mathbb{D}^{D}$, Sang Yang Lee $\mathbb{D}$, Takahiro Waki, Tomoaki Fukui $\mathbb{D}^{D}$, Keisuke Oe, \\ Tomoyuki Matsumoto ${ }^{(D}$, Takehiko Matsushita, Kotaro Nishida, Ryosuke Kuroda, \\ and Takahiro Niikura
}

\author{
Department of Orthopaedic Surgery, Kobe University Graduate School of Medicine, 7-5-1 Kusunoki-cho, Chuo-ku, \\ Kobe 650-0017, Japan \\ Correspondence should be addressed to Takahiro Niikura; tniikura@med.kobe-u.ac.jp
}

Received 2 February 2018; Revised 16 July 2018; Accepted 22 July 2018; Published 23 August 2018

Academic Editor: Werner Kolb

Copyright $\odot 2018$ Kyohei Takase et al. This is an open access article distributed under the Creative Commons Attribution License, which permits unrestricted use, distribution, and reproduction in any medium, provided the original work is properly cited.

\begin{abstract}
Rotational malreduction is a potential complication of intramedullary nailing for tibial shaft fractures. We experienced a symptomatic case of a $24^{\circ}$ externally rotated malunion that we treated with minimally invasive corrective osteotomy. A 49 -yearold man sustained a tibial shaft spiral fracture with a fibula fracture. He had been initially treated elsewhere with a reamed statically locked intramedullary nail. Bone union had been obtained, but he complained of asymmetry of his legs, difficulty walking and running, and the inability to ride a bicycle. We decided to perform corrective osteotomy in a minimally invasive fashion. After a $1 \mathrm{~cm}$ incision was made at the original fracture site, osteotomy for the affected tibia was performed with an osteotome after multiple efforts at drilling around the nail with the aim of retaining it. Fibula osteotomy was also performed at the same level. Two Kirschner wires that created an affected rotational angle between the fragments were inserted as a guide for correction. The distal locking screws were removed. Correct rotation was regained by matching the two wires in a straight line. Finally, the distal locking screws were inserted into new holes. The patient obtained bony union and has returned to his preinjury activities with no symptoms.
\end{abstract}

\section{Introduction}

Intramedullary nailing, the most common method for repairing tibial shaft fractures [1], has the advantage of requiring only minimal surgical dissection with appropriate preservation of blood supply to the fracture. The surgical implant offers biomechanical fracture stabilization and acts as a load-sharing device, allowing early postoperative mobilization [2]. Some reports, however, have described complications associated with the procedure $[3,4]$. A potentially serious, often underappreciated, complication of this procedure is rotational malreduction. In addition to presenting cosmetic dissatisfaction, torsional deformities may lead to a variety of disorders [3, 4]. For example, it has been reported that greater degrees of tibial malunion can lead to degenerative arthritis of the adjacent joint [5]. When a symptomatic rotational deformity occurs after repair using an intramedullary nail, corrective osteotomy should be done. Although several corrective surgical techniques have been reported [6-8], most require longer operation times and are more invasive. We describe a case of tibial fracture malunion treated by osteotomy while retaining the nail in a minimally invasive fashion.

\section{Case Report}

A 49-year-old man sustained a tibial shaft spiral fracture (AO/OTA classification 42-A2) with a fibular fracture (Figure 1(a)). He had no previous medical history. The fracture was treated initially at another hospital with a reamed statically locked intramedullary nail (Figure 1(b)). He noted increased external rotation of the affected leg immediately after the surgery. The tibial fracture united after a year (Figure 1(c)), but he still complained of the asymmetry of 


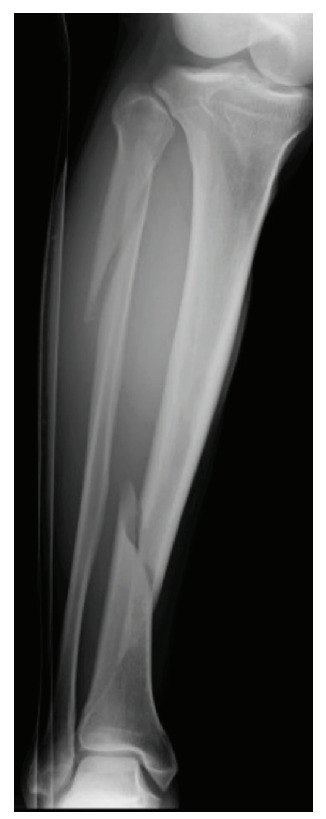

(a)

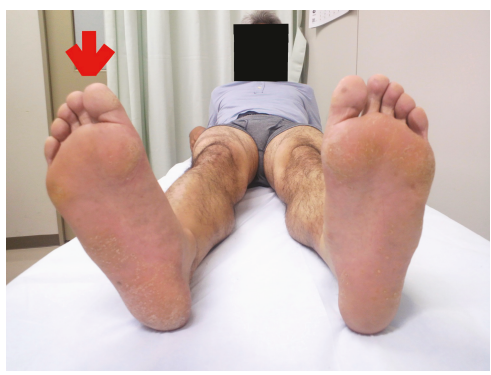

(d)

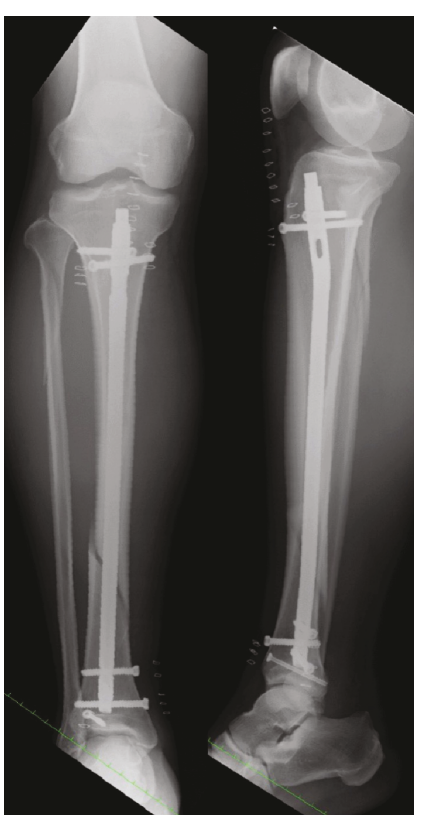

(b)

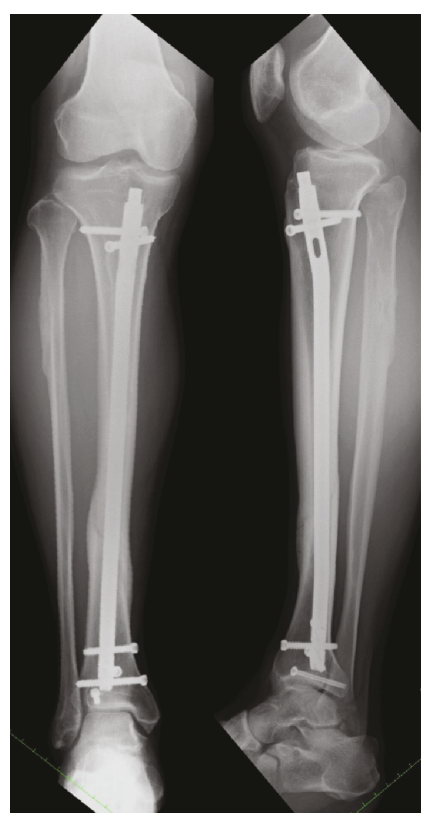

(c)

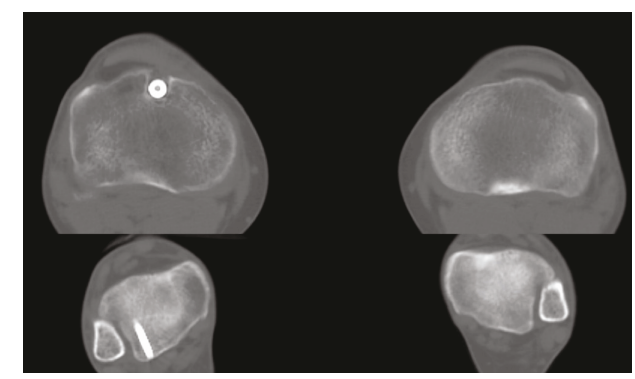

(e)

FIgURE 1: (a) Initial posttraumatic anteroposterior radiographs of the right tibia and fibula. (b-c) Anteroposterior and lateral radiographs of the right tibia obtained (b) immediate postoperatively and (c) 1 year postoperatively. (d) Photograph. (e) Computed tomography (CT) images of the right and left tibia of the patient show external tibial torsion.

his legs, difficulty walking and running, and inability to ride a bicycle. Computed tomography (CT) of both tibias showed $24^{\circ}$ of increased external rotation of the affected leg (Figures 1(d), 1(e)). Because it was a symptomatic rotational deformity, we decided to perform corrective osteotomy in a minimally invasive fashion.

The surgical procedure consisted of, first, a $1 \mathrm{~cm}$ skin incision at the original fracture site. Multiple efforts were then made to drill around the nail in a radial manner (leaving the nail in place) while using a $3.0 \mathrm{~mm}$ Kirschner wire to prepare a percutaneous osteotomy line. Osteotomy for the affected tibia was performed percutaneously using an osteotome on the prepared osteotomy line while retaining the intramedullary nail (Figure 2(a)). Fibular osteotomy was also done at the same level. Next, two $3.0 \mathrm{~mm}$ Kirschner wires, which created a $24^{\circ}$ rotational angle in the axial plane between the bone fragments, were inserted as guides for correction (Figure 2(b)). The distal locking screws were then removed. After matching the two Kirschner wires in a straight line, correct rotation was confirmed (Figure 2(c)). We assessed the rotational correction intraoperatively to evaluate both sides of the thigh-foot angle $[9,10]$. Finally, the distal three locking screws were inserted into holes different from the original hole (Figure 3(a)). At 1 year postoperatively, the patient obtained bony union and has returned to his preinjury activities with no symptoms. The implant was removed 1 year postoperatively on the patient's demand. The appropriate correction of the rotational deformity was confirmed on a CT scan (Figure 3(b)). Postoperative follow-up was continued until 5 years after the corrective osteotomy (Figure 3(c)). The patient was still free from any symptoms and had full range of hip, knee, and ankle motion.

\section{Discussion}

Tibial malrotation after intramedullary nailing is likely more common than is reported [6]. A clinical evaluation showed that it occurred with a frequency of about $0-7 \%$ $[11,12]$. A CT study reported rotational malreduction of $>10^{\circ}$ in $20-25 \%$ of patients after nailing [3]. Some studies have identified a significant malrotation difference of $>10^{\circ}$ compared with the unaffected leg $[3,13]$. Malrotation of the tibia can lead to significant limitation of function, secondary to hindfoot disability and the development of early 


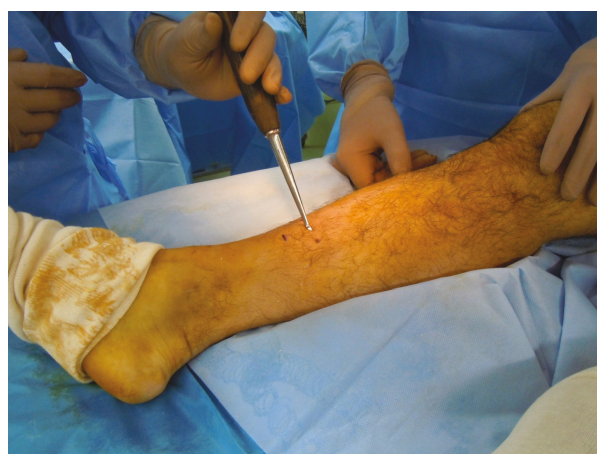

(a)

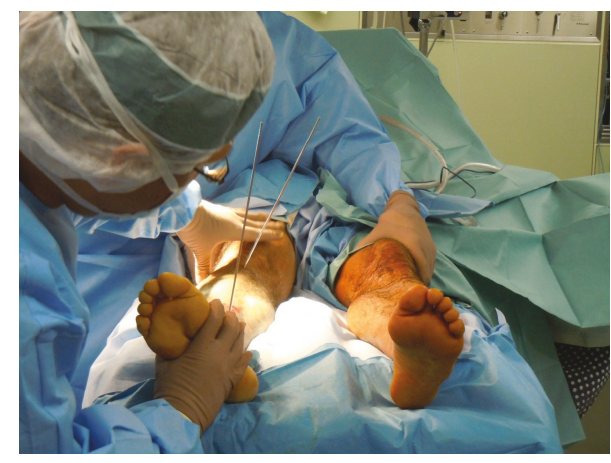

(b)

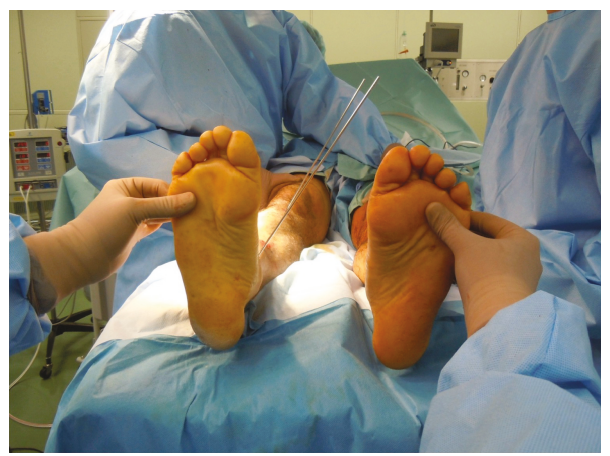

(c)

Figure 2: Photographs. (a) Small incision at the original fracture site. (b) Two $3.0 \mathrm{~mm}$ Kirschner wires were inserted as guides for correction. (c) Kirschner wires used as a guide for correcting the angle between the proximal and distal bones, which are matched in a straight line.

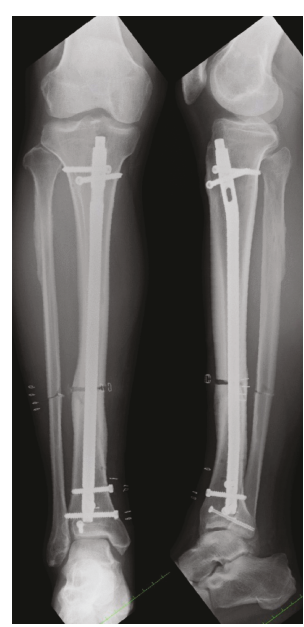

(a)

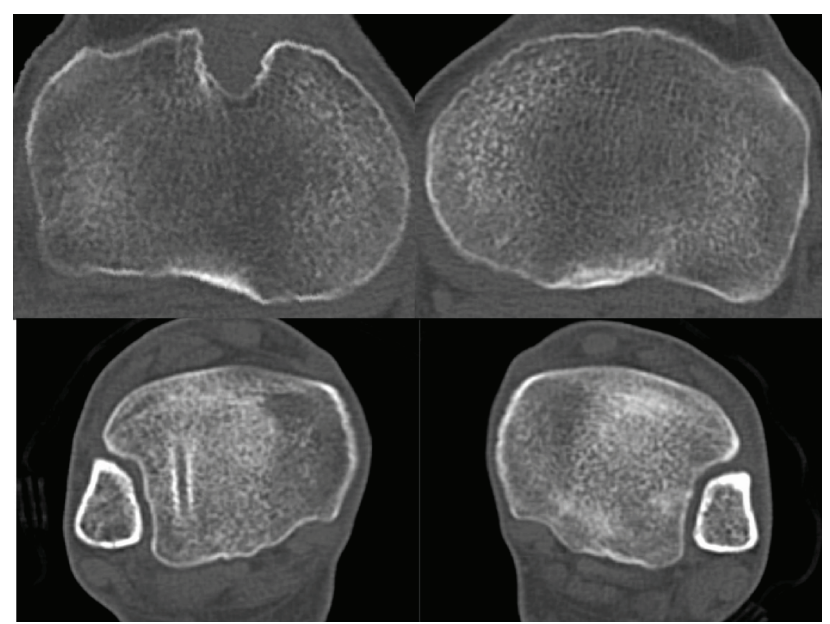

(b)

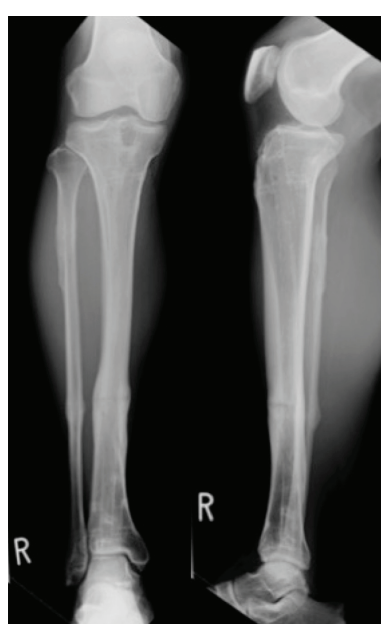

(c)

Figure 3: (a) Anteroposterior and lateral radiographs of the right tibia obtained immediately after corrective osteotomy. (b) CT images of the right and left tibias obtained 1 year after corrective osteotomy. (c) Anteroposterior and lateral radiographs of the right tibia obtained 5 years after corrective osteotomy.

degenerative arthritis [7, 14]. Although other previous reports have reported indications for corrective osteotomy, its use is still controversial [6, 7, 15]. For example, the range of acceptable malrotation is reportedly $<15^{\circ}[6]$. In contrast, another report stated that a rotational deformity of $<20^{\circ}$ did not usually produce any handicap [15]. Because our patient complained of both cosmetic (asymmetry of his legs) and functional (difficulty walking and running, inability to ride a bicycle) problems, we performed corrective surgery. Several corrective surgical techniques have been reported $[6,8,16]$. For example, after removing the intramedullary nail, a proximal (or distal) derotational osteotomy was performed and a static locked intramedullary nail placed [6]. In another, after removing the intramedullary nail, a supramalleolar derotational osteotomy was performed and plate fixation 
accomplished [16]. A third possibility is after removing the intramedullary nail, an osteotomy is performed and an Ilizarov external fixator is applied for lengthening and rotational correction [8]. For each of these techniques, however, there is a drawback: the intramedullary nail must be removed. In addition, attaching an Ilizarov external fixator takes longer to perform.

Strecker et al. have reported a technique wherein the originally implanted nail can be preserved in corrective osteotomy for rotational femoral malunion [17]. We applied this technique to tibial malrotation after intramedullary nailing because minimally invasive surgery is always the preferable option. Consequently, we obtained a good clinical result. As the surgical method described in our case can be carried out in a minimally invasive fashion, we believe it should be considered an effective option for treating tibial malrotation after nailing.

\section{Conflicts of Interest}

The authors declare that they have no conflicts of interest concerning this article.

\section{References}

[1] M. Bhandari, G. H. Guyatt, D. Tong, A. Adili, and S. G. Shaughnessy, "Reamed versus nonreamed intramedullary nailing of lower extremity long bone fractures: a systematic overview and meta-analysis," Journal of Orthopaedic Trauma, vol. 14, no. 1, pp. 2-9, 2000.

[2] B. A. Zelle and G. Boni, "Safe surgical technique: intramedullary nail fixation of tibial shaft fractures," Patient Safety in Surgery, vol. 9, no. 1, article 40, 2015.

[3] S. Puloski, C. Romano, R. Buckley, and J. Powell, "Rotational malalignment of the tibia following reamed intramedullary nail fixation," Journal of Orthopaedic Trauma, vol. 18, no. 7, pp. 397-402, 2004.

[4] A. Kyro, "Malunion after intramedullary nailing of tibial shaft fractures," Annales Chirurgiae et Gynaecologiae, vol. 86, no. 1, pp. 56-64, 1997.

[5] S. J. Svoboda, K. McHale, S. M. Belkoff, K. S. Cohen, and W. R. Klemme, "The effects of tibial malrotation on the biomechanics of the tibiotalar joint," Foot \& Ankle International, vol. 23, no. 2, pp. 102-106, 2002.

[6] K. M. Kahn and R. K. Beals, "Malrotation after locked intramedullary tibial nailing: three case reports and review of the literature," The Journal of Trauma, vol. 53, no. 3, pp. 549-552, 2002.

[7] C. van der Werken and R. K. Marti, "Post-traumatic rotational deformity of the lower leg," Injury, vol. 15, no. 1, pp. 38-40, 1983.

[8] T. Matsushita, K. Nakamura, H. Okazaki, and T. Kurokawa, "A simple technique for correction of complicated tibial deformity including rotational deformity," Archives of Orthopaedic and Trauma Surgery, vol. 117, no. 4-5, pp. 259-261, 1998.

[9] F. Ferri-de-Barros, M. Inan, and F. Miller, "Intramedullary nail fixation of femoral and tibial percutaneous rotational osteotomy in skeletally mature adolescents with cerebral palsy," Journal of Pediatric Orthopedics, vol. 26, no. 1, pp. 115-118, 2006.
[10] M. Inan, F. Ferri-de Baros, G. Chan, K. Dabney, and F. Miller, "Correction of rotational deformity of the tibia in cerebral palsy by percutaneous supramalleolar osteotomy," Journal of Bone and Joint Surgery. British Volume, vol. 87-B, no. 10, pp. 1411-1415, 2005.

[11] J. Williams, M. Gibbons, H. Trundle, D. Murray, and P. Worlock, "Complications of nailing in closed tibial fractures," Journal of Orthopaedic Trauma, vol. 9, no. 6, pp. 476-481, 1995.

[12] X. Rossignol, G. Polle, N. Bigna, and F. Dujardin, "Relationships between anatomic restitution and the situation of the fracture line in nailing procedures for distal tibial fractures," Journal of Bone and Joint Surgery. British Volume, vol. 83, pp. 41-42, 2001.

[13] M. Boucher, J. Leone, M. Pierrynowski, and M. Bhandari, "Three-dimensional assessment of tibial malunion after intramedullary nailing: a preliminary study," Journal of Orthopaedic Trauma, vol. 16, no. 7, pp. 473-483, 2002.

[14] R. M. Puno, J. J. Vaughan, M. L. Stetten, and J. R. Johnson, "Long-term effects of tibial angular malunion on the knee and ankle joints," Journal of Orthopaedic Trauma, vol. 5, no. 3, pp. 247-254, 1991.

[15] K. Yokozeki, "A study on alignment of comminuted femoral fractures treated by interlocking cylinder nailing," Kitasato Medical Journal, vol. 22, pp. 20-28, 1992.

[16] P. Selber, E. R. Filho, R. Dallalana, M. Pirpiris, G. R. Nattrass, and H. K. Graham, "Supramalleolar derotation osteotomy of the tibia, with T plate fixation: technique and results in patients with neuromascular disease," Journal of Bone and Joint Surgery. British Volume, vol. 86-B, no. 8, pp. 1170-1175, 2004.

[17] W. Strecker, P. Keppler, and L. Kinzl, "One-step corrective osteotomy for rotational femoral malunions following intramedullary nailing," Orthopaedics and Traumatology, vol. 5, pp. 200-211, 1997. 


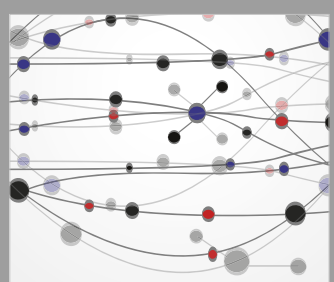

The Scientific World Journal
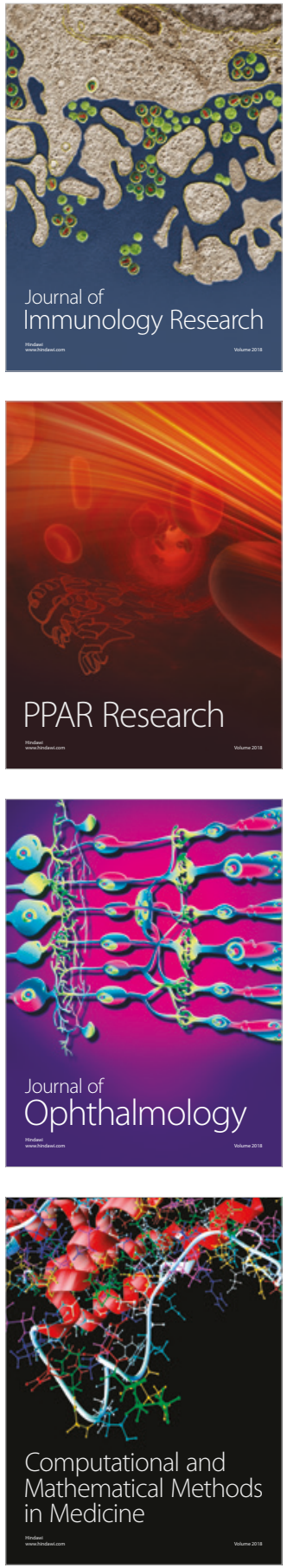

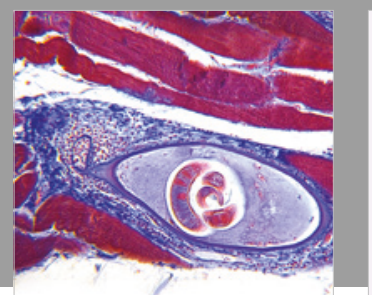

Gastroenterology Research and Practice

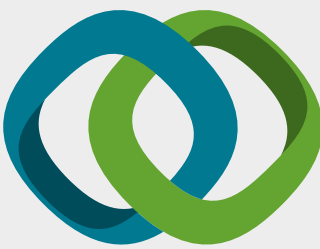

\section{Hindawi}

Submit your manuscripts at

www.hindawi.com
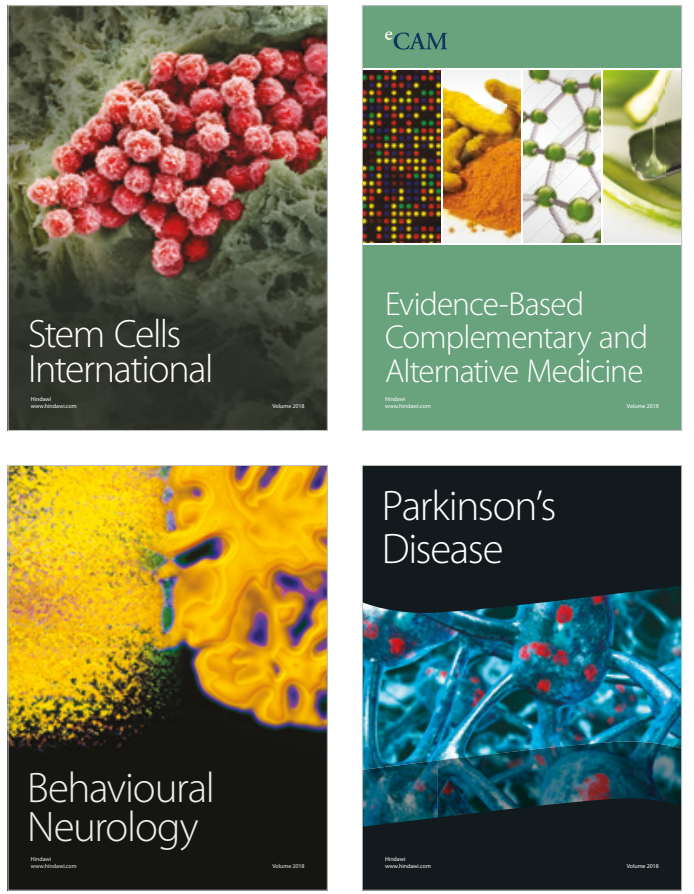

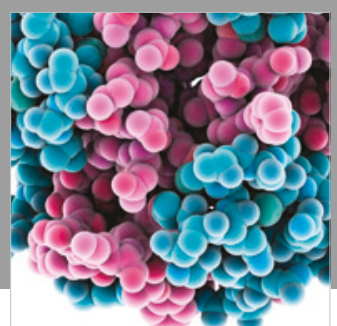

ournal of

Diabetes Research

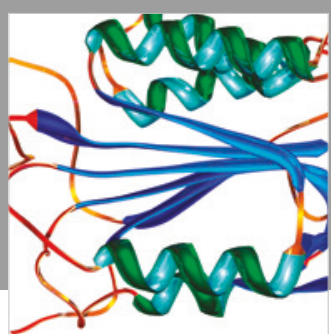

Disease Markers
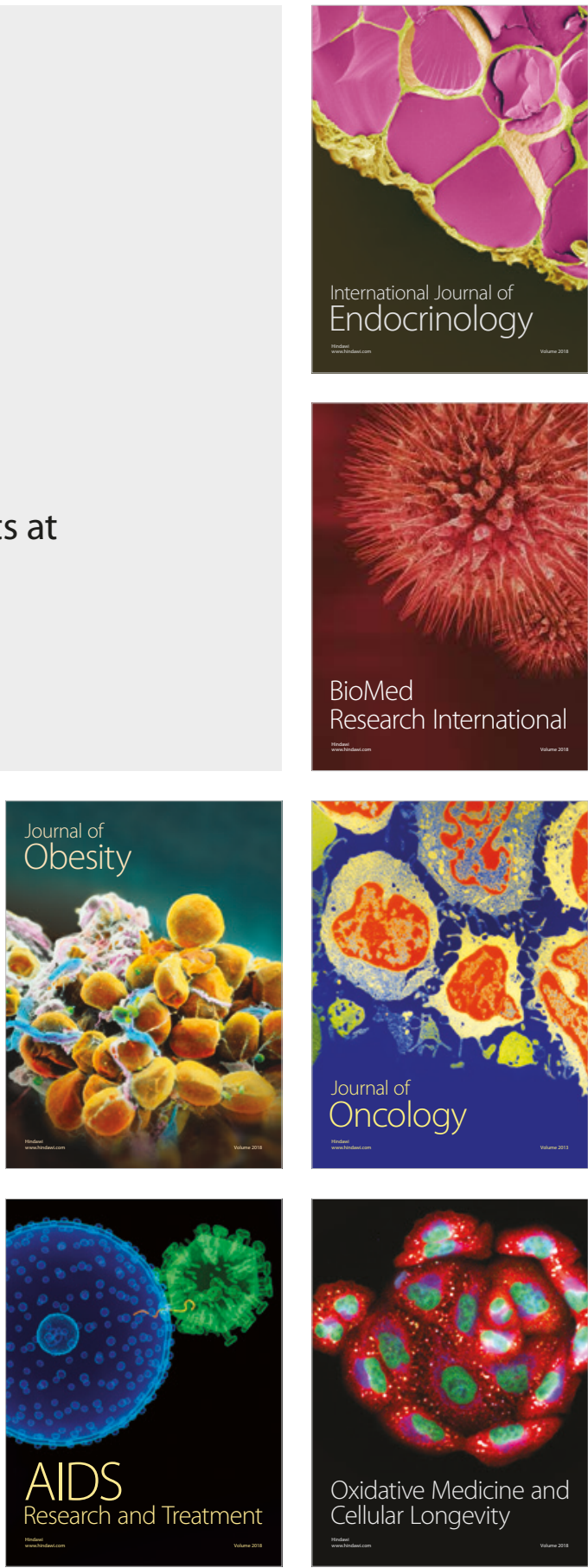